\title{
THE RELAXATION OF Mn PAIRS IN CdMnTe AT HIGH MAGNETIC FIELDS
}

\author{
T. Strutz, A.M. Witowski* AND P. Wyder \\ Max-Planck-Institut für Festkörperforschung, Hochfeld-Magnetlabor, Grenoble \\ 166X, 38042 Grenoble Cedex, France
}

\begin{abstract}
The spin-lattice relaxation in CdMnTe at $4.7 \mathrm{~K}$ has been measured at high magnetic fields up to $24.5 \mathrm{~T}$. At fields, where the ground state of nearest-neighbor exchange coupled Mn pairs changes due to energy level crossing, an increase in the relaxation rate is observed. This is attributed to the change of relaxation of nearest-neighbor Mn pairs. It proves that not only large size Mn clusters are involved in the relaxation as it has been shown previously, but also Mn pairs. It shows as well that the mixing of pair states is quite effective.
\end{abstract}

PACS numbers: 76.30.-v, 78.20.Ls, 78.47.+p

For the firsi time spin-lattice relaxation (SLR) of Mn pairs in the diluted magnetic semiconductor (DMS) CdMnTe has been observed. Our findings could directly bear on other rezent works [1, 2], concerning the width of the magnetization steps of DMS at high magnetic fields. The strongest interaction between two nearest-neighbor (n.n.) Mn ions in a DMS is the exchange interaction. The exchange Hamiltonian can be written in the Heisenberg form: $H_{\text {int }}=-2 J S_{1} \cdot S_{2}$, where $J$ is the n.n. exchange constant and $S_{1}$ and $S_{2}$ are the spin operators of two n.n. spins. Taking into account only Zeeman and exchange interactions, and neglecting all other interactions, the eigenenergies of the Mn pair at external magnetic field $B$ are given by

$$
E_{\mathrm{S}}=g \mu_{\mathrm{B}} M_{\mathrm{S}} B-J\left[S_{\mathrm{T}}\left(S_{\mathrm{T}}+1\right)-\frac{35}{2}\right],
$$

where the second term gives the energy of the multiplets with total spin $S_{\mathrm{T}}$ and magnetic quantum numbers $M_{\mathbf{S}}$. The first term describes the Zeeman splitting. The energy level scheme of these pairs is shown in the upper part of Fig. 1 (calculated for $J / k_{\mathrm{B}}=-6.5 \mathrm{~K}$ ).

We studied the SLR in $\mathrm{Cd}_{0.99} \mathrm{Mn}_{0.01} \mathrm{Te}$ at $4.7 \mathrm{~K}$ as a function of the external magnetic field up to fields of $24.5 \mathrm{~T}$ using a novel technique [3]. Ve:y short, nonresonant radiation pulses are applied on the sample. Weakly absorbed by the lattice,

*On leave from the Institute of Experimental Physics, Warsaw University, Poland. 


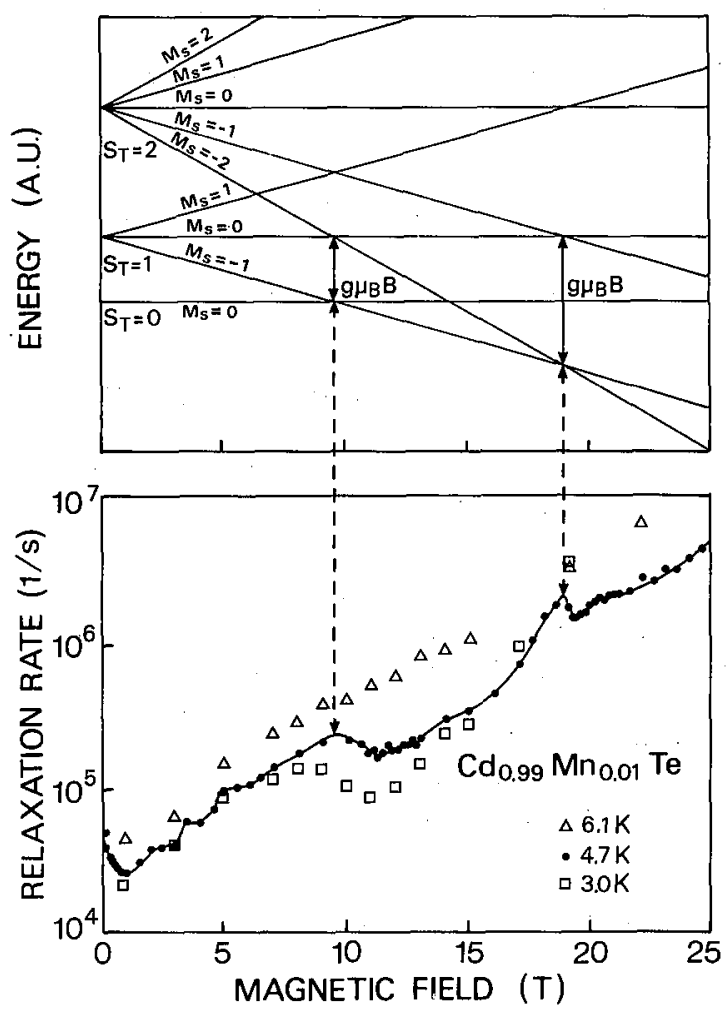

Fig. 1. Relaxation in Mn pairs. Upper part: energy level diagram of Mn pairs (see Eq. (1)). Lower part: relaxation rates of $\mathrm{Cd}_{0.99} \mathrm{Mn}_{0.01} \mathrm{Te}$ observed at $4.7 \mathrm{~K}$ as a function of the magnetic field. The line is supposed to guide the eye. At magnetic fields, where the ground state changes due to level crossing, a local increase of the SLR rate is observed, which is attributed to changes of relaxation in Mn pairs. The Zeeman splitting $g \mu_{\mathrm{B}} B$ is indicated at these field values.

they are heating the lattice only. Consequently, the spin system warms up to recover the thermal equilibrium. The SLR is observed with a pick-up coil, oriented with its axis parallel to the external field [4]. The SLR rates of $\mathrm{Cd}_{0.99} \mathrm{Mn}_{0.01} \mathrm{Te}$ measured in this way are shown in Fig. 1.

The SLR rate increases nonmonotonically with increasing field. The observed rates can be explained, especially at low fields, in terms of cross relaxation between single spins and fast relaxing spin clusters, and relaxation in these clusters [5]. Especially large size clusters consisting of three or more Mn ions, which could be $n . n$. or further nearest-neighbors, are important as fast relaxing centers. In this paper we want to focus on the local increases of the SLR rate at fields of $9.5 \mathrm{~T}$ and $19 \mathrm{~T}$. At the same field values steps in the magnetization were observed in static magnetization measurements [6-8]. At these fields the ground state of 
nearest-neighbor pairs changes due to energy level crossing. Since we observe a local increase of the SLR rate, one can conclude that at these fields, where the pair ground state changes, some relaxation processes and mechanisms are more effective than at other field values, or are even only there allowed. For example, at $9.5 \mathrm{~T}$ the Zeeman energy equals the energy separation between the ground states and the two excited states (see Fig. 1): consequently, cross relaxation to the single spins will be more effective at this field and not at other fields (resonance condition). Furthermore, the density of states of the ground state is much larger at the crossing point than at neighboring field values. Therefore, at the crossing points more pairs could participate at the relaxation of the sample magnetization.

The contribution of pairs to the SLR rate is at least in the order of $10^{5} \mathrm{~s}^{-1}$ to $10^{6} \mathrm{~s}^{-1}$ at $9.5 \mathrm{~T}$ and $19 \mathrm{~T}$ respectively, which is about $10 \%$ of the total relaxation rate at each field. On the other hand, this means that no more than $90 \%$ of the relaxation is due to other relaxation channels like cross relaxation to large size clusters or single spin relaxation at high fields as discussed in Refs. [3, 5, 9]. The relaxation rates observed by us are much faster than observed under phonon bottleneck condition at lower temperatures for other DMS, namely CdMnS and ZnMnS [2]. But particularly the relaxation of n.n. pairs is much faster than expected by Y. Shapira et al. for $\mathrm{CdMnS}$ and $\mathrm{ZnMnS}$ [1]. In these materials the magnetization steps are much narrower in pulsed fields than expected from thermal broadening. Therefore, the authors of Ref. [1] expect a pair SLR rate smaller than $2 \times 10^{3} \mathrm{~s}^{-1}$. $V$. Bindilatti et al. show [2] that these magnetization measurements are limited by a phonon bottleneck. However, in our technique based on heating the lattice by radiation pulses, phonons are provided, thus, the phonon system is slightly heated. The spin system relaxes by absorption of phonons and not by emission of phonons (as in usual relaxation experiments), until the spin system temperature reaches the phonon temperature. Therefore, the observed relaxation could not be limited by a small phonon density of states (small heat capacity of the phonons) as in the case of a phonon bottleneck [14].

The relaxation of pairs was only observed at temperatures $T \leq 4.7 \mathrm{~K}$. At higher temperatures $(T=6 \mathrm{~K})$ the SLR rates are larger and no pair effect is observed. At lower temperatures the increases are more pronounced. This is partially due to the larger population of the pair ground state at lower temperatures. Moreover, the maximum at $9.5 \mathrm{~T}$ is additionally more pronounced at low temperatures, since at fields around $11 \mathrm{~T}$ the direct relaxation process is suppressed, resulting in a total relaxation rate smaller than at neighboring fields (see Ref. [9]). It is remarkable that we see local increases in the SLR rate at temperatures higher than those temperatures at which usually the magnetization steps have been observed (0.5 K and $1.3 \mathrm{~K}$ in Ref. [8] and $2 \mathrm{~K}$ in Ref. [6]). This is not necessarily a contradiction, since we observe a change of the dynamic behavior of the spin system. On the other hand, the steps in magnetization are due to a change of a static property of the spin system, namely the magnetization.

The largest contribution to the interaction Hamiltonian between nearest-neighbor Mn pairs comes from the isotropic exchange interaction $H_{\text {int }}=-2 J S_{1} \cdot S_{2}$. The modulation of this interaction by lattice vibrations cannot lead to effective relaxation between pair states, which are eigenstates of the 
exchange Hamiltonian. Relaxation transitions are only possible as second-order effect, taking into account mixing of the states by anisotropic exchange $[10,11]$ or by hyperfine interaction $[12,13]$. Therefore, we conclude that the Mn pair states cannot be pure pair states, but they have to contain some admixtures of other states due to the mentioned interactions. In the literature also different intra-pair interactions like the Dzyaloshinski-Moriya interaction $D \cdot S_{1} \times S_{2}$ are considered $[1,13]$, providing other relaxation mechanisms.

Although one usually assumes that the relaxation in pairs is in general less effective than in larger clusters, one should expect that for CdMnTe it is still more effective than single spin relaxation at low fields, especially, because for $S$-state ions like $\mathrm{Mn}$ no efficient relaxation mechanism like the modulation of the crystalline field is known [14]. At high fields, this could be different due to the field dependence of the relaxation mechanisms [3, 9].

The authors are grateful to Prof. Dr. A. Mycielski from the Institute of Physics, Polish Academy of Sciences, for supplying the sample.

\section{References}

[1] Y. Shapira, S. Foner, D. Heiman, P.A. Wolff, C.R. McIntyre, Solid State Commun. 71, 355 (1989).

[2] V. Bindilatti, T.Q. Vu, Y. Shapira, Solid State Commun. 77, 423 (1991).

[3] T. Strutz, A.M. Witowski, P. Wyder, in Proc. 20th Int. Conf. Phys. Semicond., Thessaloniki 1990, Eds. E.M. Anastassakis, J.D. Joannopoulos, World Scientific, Singapore 1990, p. 1811.

[4] T. Strutz, A.M. Witowski, R.E.M. de Bekker, P. Wyder, Appl. Phys. Lett. 57, 831 (1990).

[5] T. Strutz, A.M. Witowski, P. Wyder, Acta Phys. Pol. A 79, 149 (1991).

[6] S. Foner, Y. Shapira, D. Heiman, P. Becla, R. Kershaw, K. Dwight, A. Wold, Phys. Rev. B 39, 11793 (1989).

[7] Y. Shapira, J. Appl. Phys. 67, 5090 (1990).

[8] Y. Shapira, N.F. Oliveira Jr., Phys. Rev. B 35, 6888 (1987).

[9] T. Strutz, Doctoral thesis, High Magnetic Field Electron Spin-Lattice Relaxation in a Diluted Magnetic Semiconductor: CdMnTe, Hartung-Gorre Verlag, Konstanz 1991.

[10] E.A. Harris, K.S. Yngvesson, J. Phys. C 1, 990 (1968); J. Phys. C 1, 1011 (1968).

[11] M.D. Glinchuk, V.G. Grachev, M.F. Deigen, Sov. Phys.-Solid State 8, 2678 (1967).

[12] L.L. Buishvili, M.D. Zviadadze, Sov. Phys.-Solid State 9, 1549 (1968).

[13] B.E. Larson, H. Ehrenreich, Phys. Rev B 39, 1747 (1989).

[14] A. Abragam, B. Bleaney, Electron Paramagnetic Resonance of Transition Ions; Clarendon Press, Oxford 1970. 